\title{
Research on the Acceptance History of the Two - Yan Words in the Song Dynasty
}

\author{
Linlin Hong ${ }^{1, \text { a }}$ \\ ${ }^{1}$ Nanchang Institute of Science \&Technology, Nanchang, Jiangxi, China, 330098
}

\section{Keywords: Yan Shu; Yan Jidao; Two Yan Words Accepted History}

\begin{abstract}
Yan Shu and Yan Jidao are of great significance to the development of poems in the Song Dynasty. The style of the poems of the two poems is slightly different, but in general, the development of graceful poems is pushed to another peak. Both of the works have indelible influence for the history of Chinese poetry and they are widely praised nearly a thousand years in China and the world. In order to understand the two words in depth, this article will discuss the life creation of Two Yan and the thought and acceptance history of his poets.
\end{abstract}

\section{Introduction}

TwoYan is the Song Dynasty famous person Yan Shu, Yan Jidao. Two Yan's poems in the history of Chinese poetry have a heavy color of the pen, so far renowned, praised for the people. Both of the words are fine and graceful, but in the style of poetry is slightly different. Yan's words are more graceful, graceful mood, more reaction to their daily thinking; Yan Jidao's nickname is abjection son, so his words reflect more depressed and sincere feelings. But the two are the same style for the style of feelings is delicate, affectionate, because the two are father-son relationship, so the style is inevitable like. In addition, the poems of Two Yan are quite controversial in the history of poems. Therefore, this paper mainly discusses the history of the poems in the Song dynasty.

\section{Research on the Deeds and Creation of Two Yan in the Song Dynasty}

Two Yan refers to Yan Shu and Yan Jidao father and son, his poems on the Song Dynasty poetry history of the contribution of extremely far, per capita in Linchuan area, it is also known as Linchuan two Yan, that is now Jiangxi. Because the father and son, so the two poetic style is very similar, it is also known as the big Yan and Xiao Yan. Many people in the Song Dynasty generally agreed that the style of Yan Jidao's poems was almost exactly the same as his father's style. The style of his father and son are divided into the recitation of small words, was later that Yan Shu words in the Song of the budding period, although Yan Jindao and his father's age gap, but by his father, and therefore inherited The little word of the father. Two Yan inherited the Southern Tang Dynasty after the main Li Yu's poetic style and rarely describe the country hatred hate, bitter hatred, and more to describe the spring flower moon, life geometry, so the word is more graceful style. However, Yan Jindao late words for the style of bias in the life of frustration, depressed unsuccessful, and his later life experience.

Yan Shu, the word with the tert, Jiangxi Linchuan people. It is said that Yan Shu from the small and intelligent, was called prodigy recommended to the holy, they won the emperor's reward and love. So Yan Shu juvenile fame, once smooth, in the future life is rare, only a few times was demoted, old age ring true. So Yan Shu's words for most of the bright and beautiful, tend to Jiangnan style. The main words for the style are divided into three categories: sentimental years, congratulations, male and female love class. Sentimental class representative is the "Huan Xisha", "magnolia flower" and so on, and more from the natural scenery associated with the life of the sense of life; male and female love class representative words such as "jade floor spring", " And so on, Yan Shu for this description is very delicate, but the word is full of sentimental sadness; congratulations, different from the above two slightly melancholy words, then the Thai people, the people live and work, so the atmosphere to write Of the lyrics for most of the song and dance Shengping, such as "more leaking child", "Jiuquan $\mathrm{Zi"}$ and so on [1]. 
Yan Jidao, word long far, Yan Shu seventh son, his birth and juvenile period should be high-spirited and the word is also the atmosphere Lingrong, talented. Later, Yan Shu was demoted, with the continuous decline of family and displaced, so the late words for the style of a great change. Yan Jidao's poems are mostly related to the song girl, Yan Jidao for some of the lower people's hardships and singer are very empathy, so his feelings for some singer, and other people have different views, famous works such as "more leaking", " Huan Xisha "and so on, which has a spread of the verse is" dance low willow floor heart, song to do peach fan at the end of the wind. "In addition to this category, Yan Jindao personal feelings and intentions of life works for many years in the experience the wind and frost after the rain made, such as "hill words".

\section{The History of the Evaluation of the Content of the Two - Yan Word in the Song Dynasty}

In fact, Yan Shu and Yan Jidao's words in the descendants of the evaluation of the type has not been high, such as the Republic of China many celebrity writers believe that Two Yan's words are mostly groaning, no meaning and so on. It can be seen at the time people's evaluation of their two is not high, or even cannot understand. After the founding of new China, the father and son of the two words is more known as Yin Yin Yan song, thought unbearable in the middle of the past few decades, few people can understand their poems. Most people think that the reference for the word is not high, so no one is interested. But perhaps in the latter part of the poetry of the two were re-understood and published works, the two poems were gradually accepted by the public, more and more people think that the words of the two Yan Wenwen delicate, the idea unique. For example, Li Shuqing that Yan Shu's poetry works compared to predecessors and it is from a number of aspects of the elaboration, there are more worthy of mining significance. And Yan Jidao's drama is also descendants of the evaluation of avant-garde thinking, pay attention to the spirit of women, the realm of elegance and so on. These evaluations for the two Yan also have a significant significance in the next few decades to effectively change the public on the evaluation of the poems of Two Yan.

In the later period, Two Yan's words are considered to have a unique charm, because of its delicate words, lyrical pen and ink, pay great attention to love, scenery, material description. For the passage of time and years of regret the same has been a lot of famous appreciation, called the two words pay attention to a word "Wan". On the use of words, many people found that the two words in common is on the "Yan", "wine", "worry" and other things are very similar. For example, there are people who comment on the contents of Two Yan "sorry and romantic", "leisure and elegant", "delicate and far-reaching" and so on, from a number of angles to clarify the difference between the two. For example, both of the verse are mainly Qingli Mingyan, for the grasp of feelings, the passing of time and the pursuit of art and so are very similar. Although the two Yan style seems similar, there are different to see, on this point, many people launched a lot of heated rebuttal. Finally, the descendants of the total return, Yan Shu for the overall mood of poetry more attention, like some of the poems in the scene and the momentum is very in place, Yan Shu advocated the beauty of things and mood to express their ideal [2]. To light rail elegant strokes depicting the fresh and elegant feelings, so that readers feel a charm. And Yan Jidao's thoughts are mostly true feelings, and magnanimous mind, its ideological content than his father since, and more rich and passionate, rhythmic, enjoys popular support, and readers can resonate, causing the reader's deep communication. However, whether it is Yan Shu's mood or Yan Jindao's sentimental sentiment today have far-reaching inspiration for the world.

\section{Study on the Acceptance History of the Two Dynasties in the Song Dynasty}

The influence of Yan Shu and Yan Jidao's poems on the history of Chinese poetry is far-reaching, and many famous verses are even in our textbooks. This side can reflect the words of Two Yan for the contribution of contemporary literature is also indelible. But as early as a thousand years of historical evolution in the process, in fact, is not so easy to be acceptable, so the acceptance of the history of the two words are divided into three main content: the general reader of the two Yan poetry acceptance history, comments The history of the acceptance of the poems of the two poets, 
and the history of the acceptance of the poets by the other poets.

The Acceptance of the General Reader for Two Yan Poetry. Most of the people for the two Yan poetry acceptance is almost the same, as early as the Song Dynasty, Yan Shu's poetic style tend to pass in the years, injury spring and autumn; Yan Jidao poetry tend to male love, emotional expression. So the styles of the two in many people's eyes are graceful sentimental sentner, following $\mathrm{Li} \mathrm{Yu}$ later. In fact, the beginning of the people for the two Yan's poetry acceptance and no later so smooth, early that the meaning of the two poems lack of connotation of new ideas, empty no flower rack without the actual content. But later in the late celebrity appreciation, more and more well, and gradually many people on the two Yan's poetic impression also has a new change, such as the current era, Two Yan's lyrics are classical poetry works, also one of the classic compulsory young people.

Critics' Acceptance of Two Yan's Poems. As early as the Northern Song Dynasty, Two Yan's poems were ignored by the literati, and even did not mind, so the words at the same time need to compile their own preface. This phenomenon shows that as early as the Song Dynasty relatively conservative era, celebrity Magi on its words and cannot accept, even worse that is a small road literature, not on the table. However, to the Southern Song Dynasty, the word is mostly sung, so Two Yan's poetry is also widely circulated, gradually more celebrities began to set off the romantic elegance of the boom, then, not only the status of the word has been recognized by the community and critics of the poems and re-placed on the table, the Southern Song Dynasty magnetic election of the four magnetic separation, Yan Jidao have been selected. The Southern Song Dynasty literati also specially for Yan Jidao works compiled a lexical and commented on the seal, we can see the late Two Yan's words for the status of a favorable upgrade.

The Acceptance of Other Poet Creators for Two Yan Poetry. Two Yan words after a few dynasties after the bumps, but also gradually harvested many poets and creators of the point of view, there are praise and derogatory. Although the poem is not on the spread of other poets, but many poets on its poetry also adopted a certain degree of acceptance of the judge. For example, Huang Tingjian that the words of the two Yan for the beautiful, can make people feel the same, Huang Tingjian and Yan Jutao good relationship, so for the "mountain word" understanding is very unique. In addition, Chao Buzhi, Li Yiyi also thinks that the words of the two Yan Qingqing, and his words are similar to the style, with words, deep rhyme law [3]. But in addition to praise, but also there is no lack of meaning, such as Li Qingzhao on the words of the two Yan: language dust, elegant enough; learn heaven, as a small lyrics, all sentences are not familiar with the ear, and often not Co-ordination, etc., enough to show her negative Two Yan poetry. And Li Qingzhao that Two Yan's poetry does not meet the standard of temperament, so it is a word is a speech.

\section{Conclusion}

In summary, from the history of the Song Dynasty to today, Two Yan's words has gradually attracted a lot of people's attention and research. However, from the historical level, the acceptance degree of the Two Yan's words is still very few. From this side it can reflect the understanding and the acceptance of the Two Yan words are also changing in thousands of years of Chinese poetry literacy. Although the poems of Yan Shu and Yan Jutao are quite controversial in accepting degree, the influence of both is very far-reaching on Chinese poetry, and it is the eternal poem of many descendants.

\section{References}

[1] Wang Huigang. Language light meaning deep love mood bitter - Yan Jutao "Nguyen Lang Gui old age residual powder like the original" analysis [J]. Famous appreciation, 2011, (27): 135-136.

[2] Zou yong. Language light love deep - Analysis of Yan Jidao "Linjiang immortal" ("Dream after the tower high lock") [J]. Language, 2014, (05): 47-48. 
[3] Gu Baolin. The Ci Poets in the Song Dynasty and the Ci Poems of the Ming Dynasty - A Study on the Spread of Yan Ou Ci [A]. Chinese Society of Ci Studies, Faculty of Letters, Shaanxi Normal University. Proceedings [C]. Chinese Society of Ci Studies, School of Liberal Arts, Shaanxi Normal University: 2010: 22. 